KYUNGPOOK Math. J. 51(2011), 261-281

http://dx.doi.org/10.5666/KMJ.2011.51.3.261

\title{
On Seifert Matrices of Symmetric Links
}

YongJu BAE* And In SoOK LeE

Department of Mathematics, College of Natural Sciences, Kyungpook National University, Daegu, 702-701, Korea

e-mail : ybae@knu.ac.kr and insooki1109@knu.ac.kr

ABSTRACT. In this paper, we will construct symmetric links by using the method adapted from the graph theory, and study a Seifert matrix of a symmetric link from the information of the Seifert matrix of the base link and the corresponding group action.

\section{Introduction}

The Seifert matrix of a link is derived from a connected Seifert surface of a link and related to the link invariants such as the signature, the nullity, the Arf invariant, the determinant, and the one-variable Alexander polynomial, see $[2][4][6][7][8][11][13]$.

A symmetric link $L$ in $\mathbb{R}^{3}$ is a link with a diagram on which a finite group can act. Figure 1 shows a link diagram, on which the Klein 4-group $\mathbb{Z}_{2} \oplus \mathbb{Z}_{2}$ can act. The periodic links of order $n$ are symmetric links whose acting group is the cyclic group $\mathbb{Z}_{n}$.

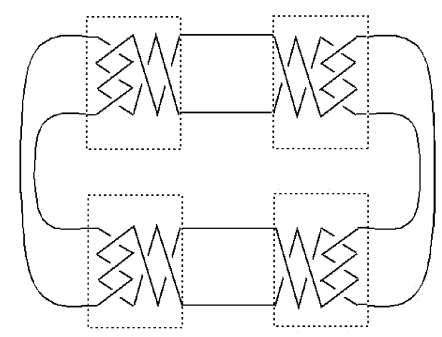

Figure 1: Symmetric link

* Corresponding Author.

Received August 23, 2010; accepted January 20, 2011.

2010 Mathematics Subject Classification: 57M25.

Key words and phrases: symmetric link, periodic link, Seifert matrix, Alexander polynomial, determinant of a link, signature of a link.

Supported by KOSEF: R01-2006-000-10944-0(2008). 
It is well-known in topological graph theory that the covering graph is constructed by a voltage assignment on the set of edges of the base graph, and that there is one-to-one correspondence between the set of all embeddings of a graph and the set of all rotation schemes. Also, by lifting the rotation scheme of a base graph to that of the covering graph, one can get the embedding of the covering graph, see [3][14].

In this paper, we will introduce a method to construct symmetric links by adapting the graph theoretical settings to link diagrams, which are 4-valent graphs embedded in $S^{2}$ with under over information, and try to calculate the Seifert matrix of such a resulting symmetric link from the information of the base link and the information of the acting group.

Theorem 1.1. Let $D$ be a diagram of a link with two fixed edges $e$ and $f$ which is depicted as in Figure 8. If $\phi: \vec{E}(D) \rightarrow G$ is a voltage assignment such that $\phi(g)=1_{G}$ for all edge $g \neq e, f$. A Seifert matrix of the symmetric link $D \times{ }_{\phi} G$ can be obtained from the Seifert matrix of $D$ and the information of the group action.

\section{Seifert matrices of links}

In this section, we will study a method to calculate a Seifert matrix of a link. In 1934, H. Seifert [12] showed how to calculate the Alexander polynomial of a link by using an orientable surface whose boundary is the given link. In fact, he gave an algorithm to produce such a surface from any link diagram, and also gave a formula for the Alexander polynomial in terms of the linking numbers of the curves on the surface. See [5] [9] for more details. A Seifert surface for an oriented link $L$ in $S^{3}$ is a connected compact oriented surface contained in $S^{3}$ which has $L$ as its boundary. We will give a brief sketch about the Seifert algorithm.

Let $D$ be a diagram of an oriented link $L$. In a small neighborhood of each crossing, make the following local change to the diagram:

Delete the crossing and reconnect the loose ends in the only way compatible with the orientation.

When this has been done at every crossing, the diagram becomes a set of disjoint simple loops in the plane. It is a diagram with no crossings. These loops are called Seifert circles. By attaching a disc to each Seifert circle and by connecting a halftwisted band at the place of each crossing of $D$ according to the crossing sign, we get a Seifert surface for $L$. The Seifert graph $\Gamma$ of $D$ is constructed as follows:

Associate a vertex with each Seifert circle and connect two vertices with an edge if their Seifert circles are connected by a twisted band.

Note that a Seifert graph $\Gamma$ is planar, and that if $D$ is connected, so do $\Gamma$. Since $\Gamma$ is a deformation retract of the Seifert surface $F$, their homology groups are isomorphic: $H_{1}(F) \cong H_{1}(\Gamma)$. Let $T$ be a spanning tree for $\Gamma$. For each edge $e \in E(\Gamma) \backslash E(T), T \cup e$ contains a unique simple closed circuit $T_{e}$, which represents 
an $1-$ cycle in $F$. The set $\left\{T_{e} \mid e \in E(\Gamma) \backslash E(T)\right\}$ of these $1-$ cycles is a homology basis for $F$. For such a circuit $T_{e}$, let $T_{e}^{+}$denote the circuit in $S^{3}$ obtained by lifting slightly along the positive normal direction of $F$. A Seifert matrix of $L$ associated to $F$ is the $n \times n$ matrix $M=\left(m_{i j}\right)$ defined by

$$
m_{i j}=l k\left(T_{e_{i}}, T_{e_{j}}^{+}\right),
$$

where $E(\Gamma) \backslash E(T)=\left\{e_{1}, \cdots, e_{n}\right\}$. The Seifert matrix of $L$ depends on the Seifert surface $F$ and the choice of generators of $H_{1}(F)$.

Let $M$ be any Seifert matrix for an oriented link $L$. The Alexander polynomial $\Delta_{L}(x) \in \mathbb{Z}\left[x^{ \pm}\right]$, the determinant $\operatorname{det}(L)$ and the signature $\sigma(L)$ of $L$ are defined by

$$
\begin{aligned}
\Delta_{L}(x) & =\operatorname{det}\left(x M-x^{-1} M^{T}\right), \\
\operatorname{det}(L) & =\left|\operatorname{det}\left(M+M^{T}\right)\right|, \\
\sigma(L) & =\sigma\left(M+M^{T}\right) .
\end{aligned}
$$

Lemma 2.1. For $e, f \in E(\Gamma) \backslash E(T), T_{e} \cap T_{f} \equiv T_{e f}$ is either empty or a simple path $v_{0} v_{1} \cdots v_{k}$ in the spanning tree $T$.

Proof. If $T_{e}$ does not meet to $T_{f}$, then $T_{e} \cap T_{f}=\emptyset$. Now, we assume that $T_{e} \cap T_{f} \neq \emptyset$. Suppose that $T_{e} \cap T_{f}$ is not connected. Let $v_{0}$ and $v_{1}$ be two vertices of $T_{e} \cap T_{f}$ which are in different components of $T_{e} \cap T_{f}$. Since $T_{e}$ and $T_{f}$ are connected, there exists a path $P_{e}$ in $T_{e}$ from $v_{0}$ to $v_{1}$ and a path $P_{f}$ in $T_{f}$ from $v_{0}$ to $v_{1}$. Then $P_{e} \neq P_{f}$, for if not, $v_{0}$ to $v_{1}$ are in the same component of $T_{e} \cap T_{f}$. A cycle $P_{e} \cup P_{f}$ is in $T_{e} \cup T_{f} \subset T$. This is a contradiction to $T$ is a tree.

If $T_{e} \cap T_{f}$ is not an empty set, let $v_{0}$ and $v_{1}$ denote two ends of $T_{e} \cap T_{f}$. Without loss of generality, we may assume that the neighborhood of $v_{0}$ looks like Figure 2. In other words, the cyclic order of edges incident to $v_{0}$ is given by $T_{e} \cap T_{f}, T_{e}, T_{f}$ with respect to the positive normal direction of the Seifert surface. Also we may assume that the directions of $T_{e}$ and $T_{f}$ are given so that $v_{0}$ is the starting point of $T_{e} \cap T_{f}$. For, if the direction is reversed, one can change the direction to adapt to our setting so that the resulting linking number changes its sign.

Theorem 2.2. For $e, f \in E(\Gamma) \backslash E(T)$, let $p$ and $q$ denote the numbers of edges in $T_{e} \cap T_{f}$ corresponding to positive crossings and negative crossings, respectively. Suppose that the local shape of $T_{e} \cap T_{f}$ in $F$ looks like Figure 2. Then,

$$
\begin{aligned}
l k\left(T_{e}, T_{f}^{+}\right) & = \begin{cases}-\frac{1}{2}(p-q), & \text { if } p+q \text { is even } ; \\
-\frac{1}{2}(p-q+1), & \text { if } p+q \text { is odd, and }\end{cases} \\
l k\left(T_{f}, T_{e}^{+}\right) & = \begin{cases}-\frac{1}{2}(p-q), & \text { if } p+q \text { is even } ; \\
-\frac{1}{2}(p-q-1), & \text { if } p+q \text { is odd } .\end{cases}
\end{aligned}
$$

Proof. If $T_{e} \cap T_{f}$ is an empty set, clearly $l k\left(T_{e}, T_{f}^{+}\right)=l k\left(T_{f}, T_{e}^{+}\right)=0$ because $T_{e}$ and $T_{f}$ are disjoint. 


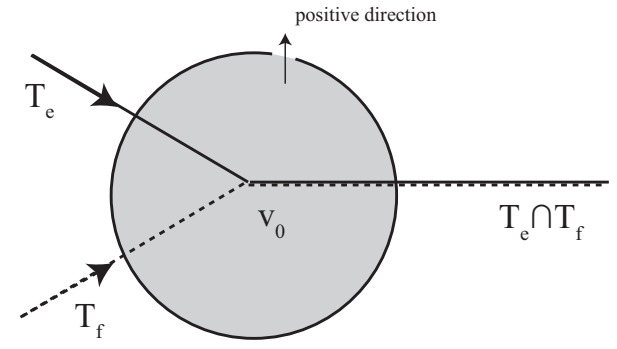

Figure 2:

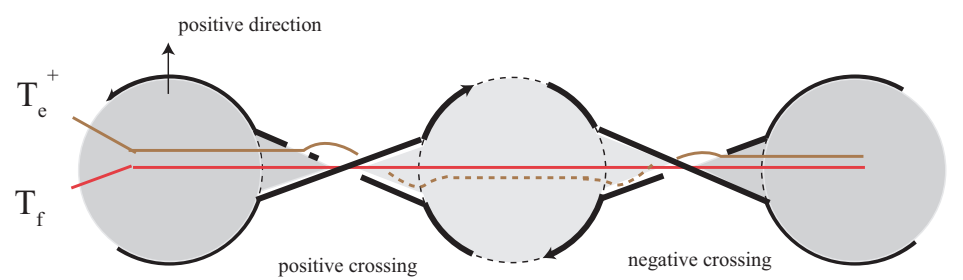

Figure 3:

Suppose that $T_{e} \cap T_{f}$ is not the empty set so that $T_{e} \cap T_{f}$ is a simple path in the spanning tree $T$. If there are two consecutive edges in $T_{e} \cap T_{f}$ whose corresponding crossings have different signs, they do not affect in calculating $l k\left(T_{e}, T_{f}^{+}\right)$ or $l k\left(T_{f}, T_{e}^{+}\right)$, as seen in Figure 3. Hence, it suffices to show that the result is true for the cases: $(p>0$ and $q=0)$ and $(p=0$ and $q>0)$.

Suppose that $p>0$ and $q=0$ and that $p$ is even. We will explain the result is true for $p=2$ and $q=0$ (see Figure 4), which is applicable for any even number $p$ and $q=0$.

From Figure 4, one can easily check that $l k\left(T_{e}, T_{f}^{+}\right)=l k\left(T_{f}, T_{e}^{+}\right)=-\frac{1}{2} p$. Note that there should be two auxiliary crossings (circled crossings) in the second picture to realize the whole paths, but they have different sign so the linking number does not change.

If $p$ is odd, we will also explain the result is true for $p=3$ and $q=0$ (see Figure 5), which is applicable for any odd number $p$ and $q=0$.

In this case, there should be an auxiliary crossing (circled crossing) in each picture to realize the whole paths, and that the auxiliary crossing in the first picture increase the linking number while the auxiliary crossing in the second picture decrease the linking number so that $l k\left(T_{e}, T_{f}^{+}\right)=l k\left(T_{f}, T_{e}^{+}\right)-1$. Indeed, for the case $p=3$ and $q=0, l k\left(T_{e}, T_{f}^{+}\right)=-2$ and $l k\left(T_{f}, T_{e}^{+}\right)=-1$.

If $p=0$ and $q>0$, one can treat the case similarly. 


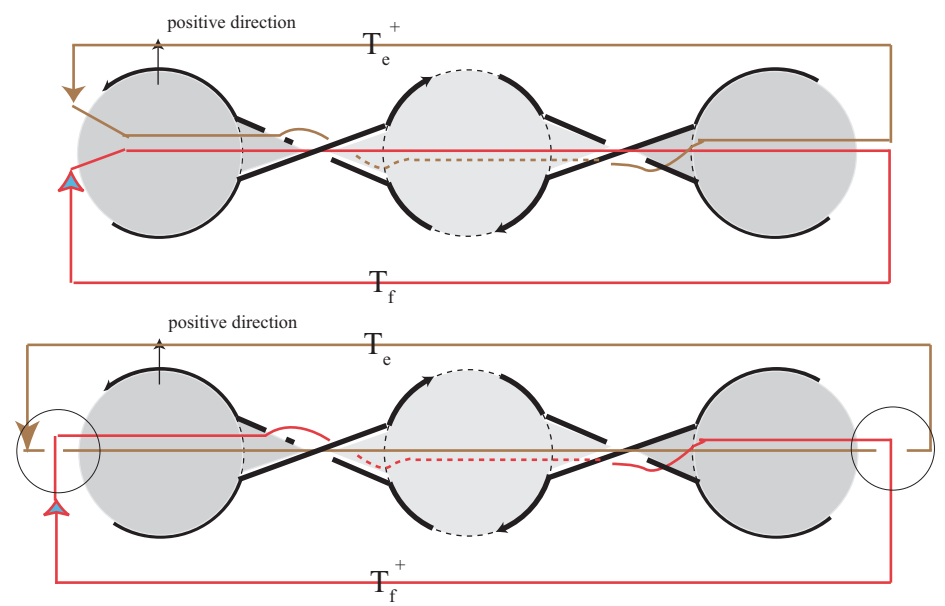

Figure 4:

Example 2.3. Let $P(3,-5,5)$ be the pretzel knot. By Theorem 2.2, we have $l k\left(e, f^{+}\right)=-\frac{1}{2}(5-0-1)=-2, l k\left(f, e^{+}\right)=l k\left(e^{+}, f\right)=-\frac{1}{2}(5-0+1)=-3$, $l k\left(e, e^{+}\right)=-\frac{1}{2}(5-3)=-1$ and $l k\left(f, f^{+}\right)=-\frac{1}{2}(5-5)=0$. Hence its Seifert matrix is

$$
M=\left(\begin{array}{rr}
-1 & -2 \\
-3 & 0
\end{array}\right)
$$

Remark 2.4. It is well known that the Seifert matrix of $P(p, q, r)$ is

$$
M=\frac{1}{2}\left(\begin{array}{cc}
p+q & q+1 \\
q-1 & q+r
\end{array}\right)
$$

where $p, q$ and $r$ be odd integers.

\section{Graph theoretical preliminary}

A graph $\Gamma=(V(\Gamma), E(\Gamma))$ consists of a finite set $V(\Gamma)$ of vertices and a finite set $E(\Gamma)$ of edges. An embedding of $\Gamma$ into a surface $F$ is a continuous injection $i: \Gamma \rightarrow F$. An embedding of $\Gamma$ into a surface $F$ is called a 2-cell embedding if each component of $F \backslash i(\Gamma)$, called a region of the embedding, is homeomorphic to the standard disc. For a vertex $v_{i} \in V(\Gamma)$, let $V\left(v_{i}\right)$ be the set of all vertices incident to $v_{i}$, and let $P_{v_{i}}: V\left(v_{i}\right) \rightarrow V\left(v_{i}\right)$ be a cyclic permutation on $V\left(v_{i}\right)$. We call $\left(P_{v_{1}} P_{v_{2}} \cdots, P_{v_{n}}\right)$ Edmond's rotation scheme. 

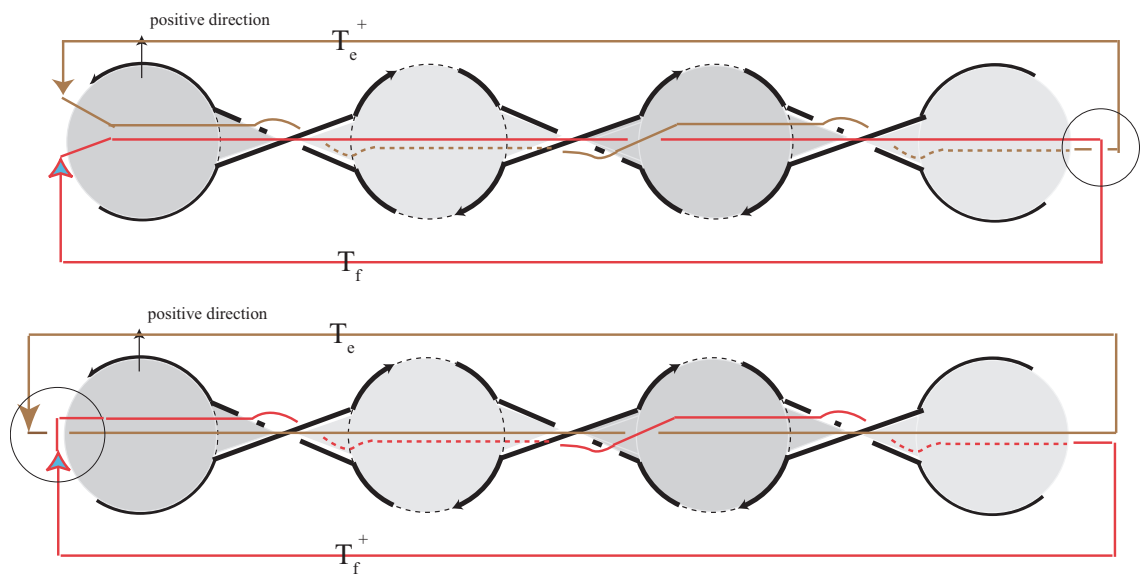

Figure 5:

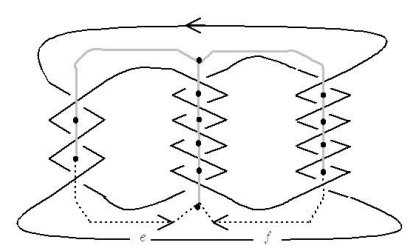

Figure 6:

Proposition 3.1([14]). A rotation scheme $\left(P_{v_{1}}, P_{v_{2}}, \cdots, P_{v_{n}}\right)$ determine a 2-cell embedding of $\Gamma$ in a surface $F$ such that there is an orientation on $F$ which is a cyclic ordering of the edge $\left[v_{i}, v_{k}\right]$ at $i$ in which the immediate successor to $\left[v_{i}, v_{k}\right]$ is $\left[v_{j}, P_{v_{i}}\left(v_{k}\right)\right]$. Conversely, for a given 2-cell embedding $i: \Gamma \rightarrow F$ in a surface $F$ with a given orientation, there is a corresponding rotation scheme $\left(P_{v_{1}}, P_{v_{2}}, \cdots, P_{v_{n}}\right)$ determining that embedding.

Let $\Gamma$ be a directed graph and $G$ a finite group. Let $D(\Gamma)$ denote the set of all directed edges of $\Gamma$ and let $\phi: D(\Gamma) \rightarrow G$ be a function, called a voltage assignment, satisfying $\phi\left(e^{-1}\right)=\phi(e)^{-1}$ for all $e \in D(\Gamma)$. We call a triple $(\Gamma, G, \phi)$ a voltage graph. The covering graph $\Gamma \times{ }_{\phi} G$ for $(\Gamma, G, \phi)$ has the vertex set $V(\Gamma) \times G$ and each edge $e=u v$ of $\Gamma$ determines the edges $(u, g)(v, g \phi(e))$ of $\Gamma \times{ }_{\phi} G$, for all $g \in G$. Notice that $\Gamma \times_{\phi} G$ is a $|G|$-fold regular covering space of $\Gamma$; in fact, every regular covering space of $\Gamma$ can be obtained in this manner.

Example 3.2. Let $\Gamma$ be a graph as in the left of Figure 7 and let $D(\Gamma)$ be the 
set of directed edges of $\Gamma$. Let $\phi: D(\Gamma) \rightarrow \mathbb{Z}_{5}$ be a voltage assignment defined by $(u, u) \rightarrow 1,(u, v) \rightarrow 0,(v, v) \rightarrow 2$, where $(u, u),(u, v),(v, v) \in D(\Gamma)$. Then, $\Gamma \times_{\phi} G$ is the graph in the right of Figure 7.
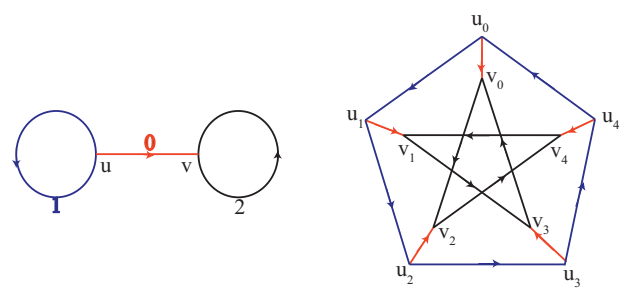

Figure 7:

The number of components of $D \times_{\phi} G$ can be calculated by the following proposition.

Proposition 3.3([14]). Let $\Gamma$ be a directed graph. Let $\phi: E(\Gamma) \rightarrow G$ be a voltage assignment. Let $H$ be the subgroup of $G$ generated by $\operatorname{Im}(\phi)$. Then the number of components of $\Gamma \times{ }_{\phi} G$ is

$$
[G: H]=|G| /|H| .
$$

Now consider a voltage graph $(\Gamma, G, \phi)$ which is 2-cell embedded in an orientable surface $S$, as described algebraically by the rotation scheme $P=\left(P_{v_{1}}, P_{v_{2}}, \ldots, P_{v_{n}}\right)$. We define the lift $\tilde{P}$ of $P$ to $\Gamma \times_{\phi} G$ as follows: if $P_{v}(v, u)=(v, w)$, then

$$
\tilde{P}_{(v, g)}((v, g),(u, g \phi(v, u)))=((v, g),(w, g \phi(v, w))),
$$

for each $g \in G$. Since $\tilde{P}=\left\{\tilde{P}_{(v, g)} \mid(v, g) \in V\left(\Gamma \times_{\phi} G\right)\right\}$ is a rotation scheme of $\Gamma \times_{\phi} G$, it determines the natural embedding of $\Gamma \times_{\phi} G$ into a surface $\tilde{S}$.

For a region $R$ of the embedding of $\Gamma$ on $S$ induced by $P$, let $|R|_{\phi}$ be the order of $\phi(\partial R)=\phi\left(e_{1}\right) \phi\left(e_{2}\right) \cdots \phi\left(e_{n}\right)$ in $G$, where $\partial R=e_{1}, e_{2}, \ldots, e_{n}$ is the ordered boundary of $R$. Since $\phi(\partial R)$ is unique up to inverses and conjugacy, $|R|_{\phi}$ is independent of the orientation of $R$ and of the initial vertex of $\partial R$. The following is well-known in topological graph theory, from which one can calculate the genus of the embedding surface $\tilde{S}$.

Proposition 3.4 $([\mathbf{1 4}])$. Let $(\Gamma, G, \phi)$ be a voltage graph with rotation schemes $P$ and $\widetilde{P}$ which determine 2-cell embeddings of $\Gamma$ and $\Gamma \times_{\phi} G$ on the orientable surfaces $S$ and $\widetilde{S}$, respectively. Then, there exists a branched covering $\rho: \widetilde{S} \rightarrow S$ such that

(1) $\rho^{-1}(\Gamma)=\Gamma \times_{\phi} G$. 
(2) $\rho \mid: \Gamma \times{ }_{\phi} G \rightarrow \Gamma$ is the graph covering map.

(3) If $b$ is a branch point of multiplicity $m$, then there exists a face $R$ in $\Gamma$ embedded in $S$ such that $b \in \operatorname{Int}(R)$ and $|R|_{\phi}=m$.

(4) If $R$ is a k-gon in $\Gamma$ embedded in $S$, then $\rho^{-1}(R)$ has $\frac{|G|}{|R|_{\phi}}$ components, each of which is a $k|R|_{\phi}$-gon region in $\Gamma \times{ }_{\phi} G \rightarrow \widetilde{S}$.

\section{Construction of symmetric links}

Let $D$ be a diagram of an oriented link $L$ embedded in $\mathbb{R}^{2} \subset S^{2}$, which can be seen as a 4-valent graph with under/over information at each vertex. Let $V, E$ and $F$ denote the numbers of vertices, edges and faces of the embedded 4 -valent graph $D$, respectively. Let $G$ be a finite group of order $n$ and $\phi: \vec{E}(D) \rightarrow G$ be a voltage assignment on a link diagram $D$, where $\vec{E}(D)$ is the set of directed edges of $D$. Let $D \times_{\phi} G \hookrightarrow \widetilde{S}$ denote the embedding of $D \times_{\phi} G$ determined by the lifted rotation scheme with $\widetilde{V}$ vertices, $\widetilde{E}$ edges and $\widetilde{F}$ faces. If the embedding surface $\widetilde{S}$ is the sphere $S^{2}$, one can obtain a symmetric link $D \times_{\phi} G$ by recovering the under/over information at each vertex according to the under/over information of the corresponding vertex of $D$. If $\widetilde{S}$ is not the sphere $S^{2}$, one may see the embedding $D \times_{\phi} G \hookrightarrow \widetilde{S}$ as a kind of virtual symmetric link.

One can calculate the genus $\widetilde{g}$ of $\widetilde{S}$, by using the definition of $D \times_{\phi} G$ and Proposition 3.4.

Theorem 4.1. Let $D$ be a connected link diagram. Let $\phi: \vec{E}(D) \rightarrow G$ be a voltage assignment. Let $H$ be the subgroup of $G$ generated by $\operatorname{Im}(\phi)$. The genus $\widetilde{g}$ of $\widetilde{S}$ is given by

$$
\widetilde{g}=|G|\left(\frac{1}{|H|}+\frac{1}{2} \sum_{R}\left(1-\frac{1}{|R|_{\phi}}\right)-1\right) .
$$

In particular, if $\widetilde{S}$ is connected, then

$$
\widetilde{g}=1-|G|+\frac{|G|}{2} \sum_{R}\left(1-\frac{1}{|R|_{\phi}}\right) .
$$

Proof. Notice that $\widetilde{V}=|G| V, \widetilde{E}=|G| E$, and $\widetilde{F}=\sum_{R} \frac{|G|}{|R|_{\phi}}$. By Proposition 3.3, $D \times{ }_{\phi} G$ has $x=[G: H]=|G| /|H|$ components. Notice that $\widetilde{S}$ has also $x$ components. By connecting all components by $x-1$ handles, one can have a connected surface $\widehat{S}$ whose genus is the same with $\widetilde{S}$. For the connected surface $\widehat{S}$, $\widehat{V}=\widetilde{V}, \widehat{E}=\widetilde{E}+(x-1)$ and $\widehat{F}=\widetilde{F}-(x-1)$. Euler's formula says that

$$
2-2 \widehat{g}=\widetilde{V}-\{\widetilde{E}+(x-1)\}+\{\widetilde{F}-(x-1)\} .
$$


Since $\widetilde{V}=|G| V, \widetilde{E}=|G| E$ and $\widetilde{F}=\sum_{R} \frac{|G|}{|R|_{\phi}}$

$$
|G|(V-E)-2 x+\sum_{R} \frac{|G|}{|R|_{\phi}}=-2 \widetilde{g} .
$$

Since $F=\sum_{R} 1$ and $V-E+F=2$, we have

$$
\widetilde{g}=x-|G|+\frac{|G|}{2} \sum_{R}\left(1-\frac{1}{|R|_{\phi}}\right) .
$$

From Theorem 4.1 and Proposition 3.3, one can see that

(1) If $\phi: \vec{E}(D) \rightarrow G$ is the constant map at the identity, then $\widetilde{S}$ is the disjoint union of $|G|$ copies of $S^{2}$.

(2) For a fixed edge $e$, if $\phi(e)=a \neq 1_{G}$ and $\phi(f)=1_{G}$ for every edge $f \neq e$, then $\widetilde{S}$ is the disjoint union of $\frac{|G|}{|a|}$ copies of $S^{2}$, where $1_{G}$ denotes the identity element of G.

Theorem 4.2. Suppose that $\phi: \vec{E}(D) \rightarrow G$ be a voltage assignment such that there are exactly two edges $e$ and $f$ with non-trivial voltages $\phi(e)=a$ and $\phi(f)=b$, and that $e$ and $f$ are on the boundary of the same region, see Figure 8. Then

(1) if $b=a^{-1} \in G$, then $\widetilde{S}$ is the disjoint union of $\frac{|G|}{|a|}$ copies of $S^{2}$.

(2) if $G=\mathbb{Z}_{p}\left(p\right.$ is prime) and $b \neq a^{-1}$, then $\widetilde{S}$ is the surface of genus $\frac{p-1}{2}$.

(3) if every element of $G$ is of order 2 , then $\widetilde{S}$ is a disjoint union of suitable copies of $S^{2}$.

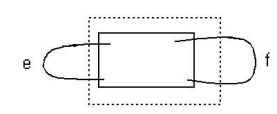

Figure 8:

Proof. By considering the three regions whose boundaries contain the fixed edges $e$ and $f$, we can get

$$
\sum_{R} \frac{1}{|R|_{\phi}}=(F-3)+\frac{1}{|a b|}+\frac{1}{|a|}+\frac{1}{|b|} .
$$


(1) If $b=a^{-1}, \sum_{R} \frac{1}{|R|_{\phi}}=(F-3)+1+\frac{2}{|a|}$. Since the number of components of $\widetilde{S}$ is $\frac{|G|}{|a|}$ by Proposition $3.3, \widetilde{g}=0$ by Theorem 4.1 .

(2) Since $p$ is prime and since $a, b$ and $a b$ are non-trivial elements, their order are $p$ so that $\sum_{R} \frac{1}{|R|_{\phi}}=(F-3)+\frac{3}{p}$. Since the number of components of $\widetilde{S}$ is 1 by Proposition $3.3, \widetilde{g}=\frac{p-1}{2}$ by Theorem 4.1 .

(3) If $b=a^{-1}$, it holds by (1). If $b \neq a^{-1}$, since every element of $G$ is of order 2 , $\sum_{R} \frac{1}{|R|_{\phi}}=(F-3)+\frac{3}{2}$. Since the number of components of $\widetilde{S}$ is $\frac{|G|}{2}$ by Proposition $3.3, \widetilde{g}=0$ by Theorem 4.1 .

As seen in the above theorem, the embedding surface $\widetilde{S}$ for $D \times{ }_{\phi} G$ may not be the sphere, in general. In the case that $\widetilde{S}$ is the sphere, the embedding of $D \times{ }_{\phi} G$ into $\widetilde{S} \cong S^{2}$ is a symmetric diagram of a link, on which the group $G$ can act. For the case that $\widetilde{S}$ is not the sphere, one can see the embedding of $D \times{ }_{\phi} G$ into $\widetilde{S}$ as a symmetric virtual diagram of a virtual link, on which the group $G$ can act.

Example 4.3. Let $D$ be a diagram as in the left of Figure 9, which is the denominator of the rational tangle $C(3,-2)$. Let $G$ be the Klein 4-group $\mathbb{Z}_{2} \oplus \mathbb{Z}_{2}$. Consider the voltage assignment $\phi: \vec{E}(D) \rightarrow G$ given by $\phi(e)=(1,0)$ and $\phi(f)=(0,1)$ as in the left of Figure 9. By Theorem 4.2, the embedding surface $\widetilde{S}$ for $D \times_{\phi} G$ is the sphere $S^{2}$. In fact, the symmetric diagram $D \times{ }_{\phi} G$ obtained by our construction is given in the right of Figure 9.

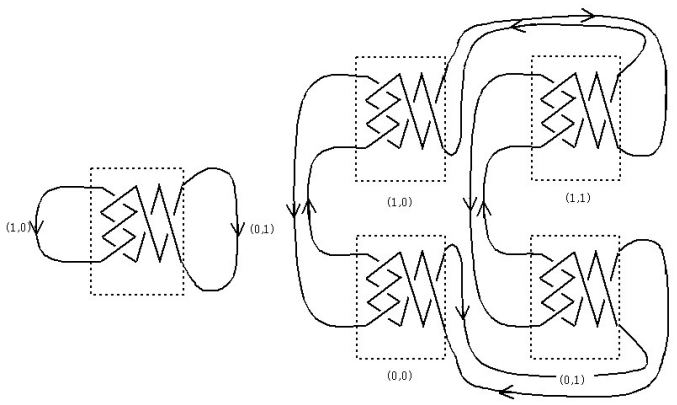

Figure 9: 


\section{Seifert matrices of symmetric links}

In this section, we will try to find a Seifert matrix of a symmetric link $D \times{ }_{\phi} G$, in the case that the corresponding embedding surface is the sphere.

If $G$ is trivial, clearly $D \times_{\phi} G$ is $D$, and hence a Seifert matrix of $D \times_{\phi} G$ equals to a Seifert matrix of $D$. From now on, we assume that $G$ is a non-trivial group.

Lemma 5.1. Suppose that $\phi: \vec{E}(D) \rightarrow G$ is the constant function at $1_{G}$. Then a Seifert matrix $\widetilde{M}$ of $D \times_{\phi} G$ has the form;

$$
\widetilde{M} \cong\left(\bigoplus_{|G|} M\right) \bigoplus O_{|G|-1}=\left(\begin{array}{ccccc}
M & O & \cdots & O & O \\
O & M & \cdots & O & O \\
\vdots & \vdots & \ddots & \vdots & \vdots \\
O & O & \cdots & O & O
\end{array}\right),
$$

where $|G|$ denotes the order of $G$ and $O$ is the zero matrix.

Proof. From Theorem 4.1 and Proposition 3.3, one can see that $D \times{ }_{\phi} G$ is the disjoint union of $|G|$ copies of $D$, which is embedded in the disjoint union of $|G|$ copies of $S^{2}$.

Corollary 5.2. Suppose that $\phi: \vec{E}(D) \rightarrow G$ is the constant function at $1_{G}$. Then $\operatorname{det}\left(D \times_{\phi} G\right)=0, \sigma\left(D \times_{\phi} G\right)=|G| \sigma(L)$ and $\Delta\left(D \times_{\phi} G\right)=0$.

Proof. It is trivial by definitions of the determinant, the signature, and the Alexander polynomial of $L$.

Lemma 5.3. Suppose that there is one edge e such that $\phi(e)=a \neq 1_{G}$ and $\phi\left(e^{\prime}\right)=1_{G}$ for all $e^{\prime} \neq e$. Then a Seifert matrix $\widetilde{M}$ of $D \times_{\phi} G$ has the form:

$$
\begin{aligned}
\widetilde{M} \cong & \left(\bigoplus_{|G|} M\right) \bigoplus O_{\frac{|G|}{|a|}-1} \\
= & \left(\begin{array}{ccccc}
M & O & \cdots & O & O \\
O & M & \cdots & O & O \\
\vdots & \vdots & \ddots & \vdots & \vdots \\
O & O & \cdots & O & O
\end{array}\right) .
\end{aligned}
$$

Proof. From Theorem 4.1 and Proposition 3.3, one can see that $D \times_{\phi} G$ is the disjoint union of $\frac{|G|}{|a|}$ copies of the connected sum $D \sharp D \sharp \cdots \sharp D$ (|a| times), which is embedded in the disjoint union of $\frac{|G|}{|a|}$ copies of $S^{2}$.

Corollary 5.4. Suppose that there is one edge $e$ such that $\phi(e)=a \neq 1_{G}$ and $\phi\left(e^{\prime}\right)=1_{G}$ for all $e^{\prime} \neq e$. Then 
(1) if a is a generator of $G$, then $\operatorname{det}\left(D \times_{\phi} G\right)=\operatorname{det}(L)^{|G|}, \sigma\left(D \times_{\phi} G\right)=|G| \sigma(L)$ and $\Delta\left(D \times{ }_{\phi} G\right)=\Delta(L)^{|G|}$.

(2) if $a$ is not a generator of $G$, then $\operatorname{det}\left(D \times{ }_{\phi} G\right)=0, \sigma\left(D \times{ }_{\phi} G\right)=|G| \sigma(L)$ and $\Delta\left(D \times_{\phi} G\right)=0$.

Proof. (1) If $a$ is a generator of $G, \frac{|G|}{|a|}=1$. By Lemma 5.3, $\widetilde{M} \cong \oplus_{|G|} M$. By definitions of the determinant, the signature, and the Alexander polynomial of $L$, $\operatorname{det}\left(D \times_{\phi} G\right)=\operatorname{det}(L)^{|G|}, \sigma\left(D \times_{\phi} G\right)=|G| \sigma(L)$ and $\Delta\left(D \times_{\phi} G\right)=\Delta(L)^{|G|}$.

(2) If $a$ is not a generator of $G$, then $\frac{|G|}{|a|}>1$ so, by Lemma $5.3, \operatorname{det}\left(D \times_{\phi} G\right)=0$, $\sigma\left(D \times_{\phi} G\right)=|G| \sigma(L)$ and $\Delta\left(D \times_{\phi} G\right)=0$.

Now suppose that there exist two edges $e, f$ in $D$ such that $\phi(e)=a \neq$ $1_{G}, \phi(f)=b \neq 1_{G}$ and $\phi(g)=1_{G}$ for every edge $g \neq e, f$.

Without loss of generality, we may assume that $e$ and $f$ are contained in different Seifert circles. For, if they are contained in the same Seifert circle, by applying Reidemeister move II, we can change $D$ into $D^{\prime}$ so that the corresponding edges are contained in different Seifert circles, as seen in Figure 10. It is easy to check that $D \times{ }_{\phi} G$ and $D^{\prime} \times{ }_{\phi} G$ are the diagrams of the same link and that the Seifert matrix of $D \times{ }_{\phi} G$ equals to that of $D^{\prime} \times{ }_{\phi} G$.

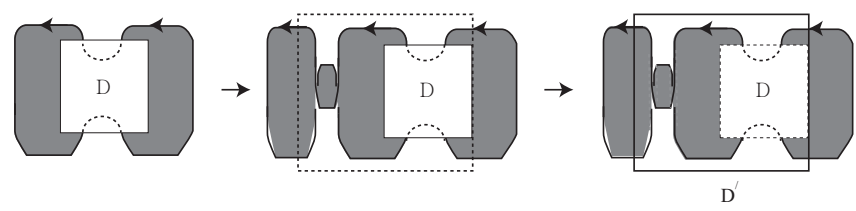

Figure 10:

Let $F$ be a Seifert surface obtained from $D$ by the Seifert algorithm and $\Gamma$ its Seifert graph. Let $T$ be a spanning tree of $\Gamma$ and let $\langle a, b\rangle$ denote the subgroup of $G$ generated by $a$ and $b$. From Proposition 3.1 and 3.4, we know that

(1) $\rho^{-1}(T)$ is a $|G|$-fold branched covering of $T$.

(2) $D \times_{\phi} G$ has $c=\frac{|G|}{|\langle a, b\rangle|}$ components, say $\widetilde{D_{1}}, \widetilde{D_{2}}, \cdots, \widetilde{D_{c}}$.

(3) $\rho^{-1}(T)$ has also $c$ components $\widetilde{T_{1}}, \widetilde{T}_{2}, \cdots, \widetilde{T}_{c}$, each of them is a spanning tree of the corresponding component.

(4) $\widetilde{T}_{i}$ is a $|\langle a, b\rangle|$-fold branched covering of $T$ for all $i$.

From now on, we will try to find the set of generators of $H_{1}(\widetilde{F})$ of the Seifert surface $\widetilde{F}$ of $D \times_{\phi} G$. First of all, we set the voltage group $G$ by $G=\left\{g_{1}, g_{2}, \cdots, g_{n}\right\}$. 
For each edge $a$, we denote $\rho^{-1}(a)$ by $\left\{a^{g_{1}}, a^{g_{2}}, \cdots, a^{g_{n}}\right\}$. Let $u$ and $v$ be the vertices in the Seifert graph $\Gamma$ correspond to the Seifert circles containing $e$ and $f$, respectively. Since $\Gamma$ is connected, the spanning tree $T$ of $\Gamma$ is also connected. Hence there is a unique path $P$ from $u$ to $v$ contained in $T$. Note that $\rho^{-1}(P)$ is a branched covering of $P$ with exactly two branch points $u$ and $v$, and that $\rho^{-1}(P)$ has $c$ components $\widetilde{P_{1}}, \widetilde{P_{2}}, \cdots, \widetilde{P_{c}}$.

If the length of $P$ is $l$, the number of the vertices of $\widetilde{P}_{i}$ is $(l-1)|\langle a, b\rangle|+\frac{|\langle a, b\rangle|}{|a|}+$ $\frac{|\langle a, b\rangle|}{|b|}$ so that the number of edges in a spanning tree of $\widetilde{P}_{i}$ is given by $(l-1)|\langle a, b\rangle|+$ $\frac{|\langle a, b\rangle|}{|a|}+\frac{|\langle a, b\rangle|}{|b|}-1$. Hence the number of edges in $\widetilde{P}_{i}$ which is not in a spanning tree is $l|\langle a, b\rangle|-\left\{(l-1)|\langle a, b\rangle|+\frac{|\langle a, b\rangle|}{|a|}+\frac{|\langle a, b\rangle|}{|b|}-1\right\}=|\langle a, b\rangle|-\frac{|\langle a, b\rangle|}{|a|}-\frac{|\langle a, b\rangle|}{|b|}+1$.

Since $\rho^{-1}(P)$ has $\frac{|G|}{\langle a, b\rangle \mid}$ components, the number of edges in $\rho^{-1}(P)$ not in a spanning tree is $k=|G|\left(\frac{1}{|\langle a, b\rangle|}-\frac{1}{|a|}-\frac{1}{|b|}+1\right)$. In other word, for a spanning tree $T$ of $\Gamma, \rho^{-1}(T)$ is not a spanning tree of $\rho^{-1}(\Gamma)$, in general. But, by removing $|G|\left(\frac{1}{\langle\langle a, b\rangle|}-\frac{1}{|a|}-\frac{1}{|b|}+1\right)$ edges from $\rho^{-1}\left(e_{0}\right)$ for a fixed edge $e_{0}$ in $P$, we can get a spanning tree $\widetilde{T}$ of $\rho^{-1}(\Gamma)$.

Since for each edge $e^{\prime}$ of $\Gamma$, not in $T, \rho^{-1}\left(e^{\prime}\right)$ consists of $|G|$ edges of $\rho^{-1}(\Gamma)$, not in $\widetilde{T}$. Hence the number of generators of $\rho^{-1}(\Gamma)$ is

$$
|G| \times\left(\text { the number of generators of } \Gamma+\frac{1}{|\langle a, b\rangle|}-\frac{1}{|a|}-\frac{1}{|b|}+1\right) .
$$

Let $\left\{a_{1}, a_{2}, \cdots, a_{m}\right\}$ denote the set of generators of $H_{1}(F)$. Since $a_{i}$ is not in the spanning tree $T$ of $\Gamma,\left\{a_{i}^{g_{j}} \mid i=1, \cdots, m, j=1, \cdots, n\right\}$ is a set of edges of the Seifert graph $\rho^{-1}(\Gamma)$ of $D \times_{\phi} G$ which are not in the spanning tree $\widetilde{T}$.

For the fixed edge $e_{0}$ in $P$, by choosing $k=|G|\left(\frac{1}{|\langle a, b\rangle|}-\frac{1}{|a|}-\frac{1}{|b|}+1\right)$ edges, say $\left\{e_{0}^{g_{1}}, e_{0}^{g_{1}}, \cdots, e_{0}^{g_{k}}\right\}$, from $\rho^{-1}\left(e_{0}\right)$, we can obtain all other generators of $H_{1}(\widetilde{F})$. The set of all generators of $H_{1}(\widetilde{F})$ is given by

$$
\left\{a_{i}^{g_{j}} \mid i=1, \cdots, m, j=1, \cdots, n\right\} \cup\left\{e_{0}^{g_{1}}, e_{0}^{g_{2}}, \cdots, e_{0}^{g_{k}}\right\} .
$$

Example 5.5. For the diagram with the voltage assignment in Example 4.3, the number of generators of its Seifert graph is 2, while the number of components of $D \times_{\phi} G$ is 1 because $|G|=\left|\mathbb{Z}_{2} \oplus \mathbb{Z}_{2}\right|=4$ and $|\langle a, b\rangle|=|\langle(1,0),(0,1)\rangle|=4$. Since $|a|=|b|=2$, the number of generators of the Seifert surface of $D \times_{\phi} G$ is $4\left(2+\frac{1}{4}-\frac{1}{2}-\frac{1}{2}+1\right)=9$. One can see the generators of the Seifert surface of $D \times_{\phi} G$ at Figure 11.

To calculate of the linking numbers between two generators of the Seifert Surface of $D \times_{\phi} G$, we need to see through the Seifert graph of the base diagram. For $a \in E(\Gamma) \backslash E(T)$, there exists an unique path in $T$ whose union with the edge $a$ gives a simple closed circuit $T_{a}$.

For the path $P$ between two end vertices $u$ and $v$ with $p$ positive crossings and $q$ negative crossings, we construct an auxiliary closed path $\bar{P}$ as follows: 

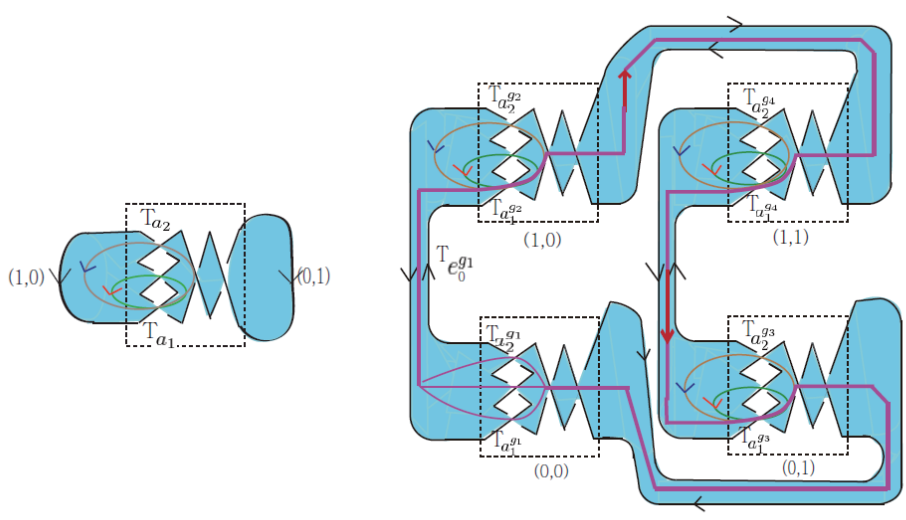

Figure 11:

- Identify $u$ and $v$ if $p+q$ is even,

- Connect $u$ and $v$ by an auxiliary edge with positive sign if $p+q$ is odd.

Notice that $\bar{P}$ has even number of edges, and that $l k\left(\bar{P}, \bar{P}^{+}\right)=-\frac{1}{2}(p-q)$ if $p+q$ is even, while $l k\left(\bar{P}, \bar{P}^{+}\right)=-\frac{1}{2}(p-q+1)$ if $p+q$ is odd.

The calculation of the linking numbers between two generators of $\widetilde{F}$ depends on the relation between the fixed edge $e_{0}$ and the corresponding generators of $F$. The formulae in Lemma 5.6 have the key role in the calculation of the linking numbers.

From now on, for a generator $a_{i}$ of $H_{1}(F)$, let $T_{a_{i}}^{c}$ denote the simple closed path obtained from $\bar{P}$ by removing $T_{a_{i}}$ and then adding the edge $a_{i}$, see Figure 12 .

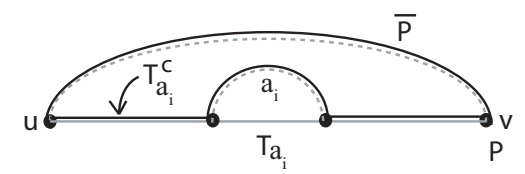

Figure 12:

In the following lemmas(Lemma 5.6-Lemma 5.11), let $e$ and $f$ denote the edges in $E(\Gamma) \backslash E(T)$ such that $T_{e}$ and $T_{f}$ are depicted in Figure 2.

The following Lemma is about the linking numbers in curves on the Seifert surface of the base link, which gives an important tool for the calculation of the 
linking numbers of generators of $\widetilde{F}$.

Lemma 5.6. Suppose that $T_{e}$ and $T_{f}$ have a common edge with $P$, respectively, and that $T_{e} \backslash\{e\}$ contains $p_{e}$ positive crossings and $q_{e}$ negative crossings and $T_{f} \backslash\{f\}$ contains $p_{f}$ positive crossings and $q_{f}$ negative crossings. Then

(1) if $e_{0} \in T_{e} \cap T_{f}$,

$$
\begin{aligned}
& l k\left(T_{f}^{c}, T_{e}^{c+}\right)=l k\left(\bar{P}, \bar{P}^{+}\right)+l k\left(T_{f}, T_{e}^{+}\right)+\frac{1}{2}\left\{\left(p_{e}+p_{f}\right)-\left(q_{e}+q_{f}\right)\right\} \\
& l k\left(T_{e}^{c}, T_{f}^{c+}\right)=l k\left(\bar{P}, \bar{P}^{+}\right)+l k\left(T_{e}, T_{f}^{+}\right)+\frac{1}{2}\left\{\left(p_{e}+p_{f}\right)-\left(q_{e}+q_{f}\right)\right\} .
\end{aligned}
$$

(2) if $e_{0} \in T_{f}$ and if $e_{0} \notin T_{e}$,

$$
l k\left(T_{f}^{c}, T_{e}^{+}\right)=\frac{1}{2}\left(p_{e}-q_{e}-1\right) \text { and } l k\left(T_{e}, T_{f}^{c+}\right)=\frac{1}{2}\left(p_{e}-q_{e}+1\right) .
$$

(3) if $e_{0} \notin T_{f}$ and if $e_{0} \in T_{e}$,

$$
l k\left(T_{f}, T_{e}^{c+}\right)=\frac{1}{2}\left(p_{f}-q_{f}-1\right) \text { and } l k\left(T_{e}^{c}, T_{f}^{+}\right)=\frac{1}{2}\left(p_{f}-q_{f}+1\right) .
$$

Proof. Suppose that $P$ contains $p$ positive crossings and $q$ negative crossings. Note that $T_{e} \cap T_{f}$ and $T_{e}^{c} \cap T_{f}^{c}$ are simple paths. Let $p_{e f}$ and $q_{e f}$ denote the numbers of positive edges and negative edges in $T_{e} \cap T_{f}$. Then $T_{e}^{c} \cap T_{f}^{c}$ consists of $p-p_{e}-p_{f}+p_{e f}$ positive crossings and $q-q_{e}-q_{f}+q_{e f}$ negative crossings if $p+q$ is even, while it consists of $(p+1)-p_{e}-p_{f}+p_{e f}$ positive crossings and $q-q_{e}-q_{f}+q_{e f}$ negative crossings if $p+q$ is odd.

(1) If $p+q$ is even and if $p_{e f}+q_{e f}$ is even, then $T_{e}^{c} \cap T_{f}^{c}$ consists of even number of edges because $p_{e}+q_{e}$ and $p_{f}+q_{f}$ are odd. Hence, by Theorem 2.2,

$$
\begin{aligned}
l k\left(T_{f}^{c}, T_{e}^{c+}\right) & =-\frac{1}{2}\left(\left(p-p_{e}-p_{f}+p_{e f}\right)-\left(q-q_{e}-q_{f}+q_{e f}\right)\right) \\
& =-\frac{1}{2}(p-q)+\frac{1}{2}\left(p_{e}-q_{e}\right)+\frac{1}{2}\left(p_{f}-q_{f}\right)-\frac{1}{2}\left(p_{e f}-q_{e f}\right) \\
& =l k\left(\bar{P}, \bar{P}^{+}\right)+l k\left(T_{f}, T_{e}^{+}\right)+\frac{1}{2}\left\{\left(p_{e}+p_{f}\right)-\left(q_{e}+q_{f}\right)\right\}, \\
l k\left(T_{e}^{c}, T_{f}^{c+}\right) & =l k\left(\bar{P}, \bar{P}^{+}\right)+l k\left(T_{e}, T_{f}^{+}\right)+\frac{1}{2}\left\{\left(p_{e}+p_{f}\right)-\left(q_{e}+q_{f}\right)\right\} .
\end{aligned}
$$

If $p+q$ is even and if $p_{e f}+q_{e f}$ is odd, then $T_{e}^{c} \cap T_{f}^{c}$ consists of odd number of 
edges because $p_{e}+q_{e}$ and $p_{f}+q_{f}$ are odd. By Theorem 2.2,

$$
\begin{aligned}
l k\left(T_{f}^{c}, T_{e}^{c+}\right) & =-\frac{1}{2}\left(\left(p-p_{e}-p_{f}+p_{e f}\right)-\left(q-q_{e}-q_{f}+q_{e f}\right)-1\right) \\
& =-\frac{1}{2}(p-q)+\frac{1}{2}\left(p_{e}-q_{e}\right)+\frac{1}{2}\left(p_{f}-q_{f}\right)-\frac{1}{2}\left(p_{e f}-q_{e f}-1\right) \\
& =l k\left(\bar{P}, \bar{P}^{+}\right)+l k\left(T_{f}, T_{e}^{+}\right)+\frac{1}{2}\left\{\left(p_{e}+p_{f}\right)-\left(q_{e}+q_{f}\right)\right\} \\
l k\left(T_{e}^{c}, T_{f}^{c+}\right), & =l k\left(\bar{P}, \bar{P}^{+}\right)+l k\left(T_{e}, T_{f}^{+}\right)+\frac{1}{2}\left\{\left(p_{e}+p_{f}\right)-\left(q_{e}+q_{f}\right)\right\} .
\end{aligned}
$$

For the case that $p+q$ is odd, $T_{e}^{c} \cap T_{f}^{c}$ contains $(p+1)-p_{e}-p_{f}+p_{e f}$ positive crossings and $q-q_{e}-q_{f}+q_{e f}$ negative crossings. By the similar argument, one can obtain the required equations.

(2) Notice that $T_{f}^{c}$ and $T_{e}$ contain $p_{e}$ positive crossings and $q_{e}$ negative crossings in common, and that they have the opposite direction. Since $T_{f}{ }^{c} \cap T_{e}$ consists of $p_{e}+q_{e}$ edges and $p_{e}+q_{e}$ is odd, by Theorem 2.2,

$$
l k\left(T_{f}^{c}, T_{e}^{+}\right)=\frac{1}{2}\left(p_{e}-q_{e}-1\right) \text { and } l k\left(T_{e}, T_{f}^{c+}\right)=\frac{1}{2}\left(p_{e}-q_{e}+1\right) .
$$

(3) Similar with (2).

The calculation of the linking numbers between two generators of the Seifert surface of $D \times_{\phi} G$ depends on the relationship between the lifts $e_{0}^{g_{1}}, e_{0}^{g_{2}}, \cdots, e_{0}^{g_{k}}$ of the fixed edge $e_{0}$, too. We will give formulae for the calculation case by case.

Lemma 5.7. Suppose that $P$ contains $p$ positive crossings and $q$ negative crossings and that $T_{e}$ and $T_{f}$ have a common edge with $P$, respectively. For $\alpha$ with $1 \leq \alpha \leq k$ and hence $e_{0}^{g_{\alpha}} \notin E(\widetilde{T})$,

(1) if $e_{0} \in T_{e} \cap T_{f}$, then

$$
\begin{aligned}
& l k\left(T_{f^{g_{\alpha}}}, T_{e^{g_{\alpha}}}+\right)= \begin{cases}(d-1) l k\left(\bar{P}, \bar{P}^{+}\right)+l k\left(T_{f}^{c}, T_{e}^{c+}\right), & \text { if } p+q \text { is even } \\
(d-1) l k\left(\bar{P}, \bar{P}^{+}\right)+l k\left(T_{f}^{c}, T_{e}^{c+}\right)+\frac{1}{2} d, & \text { if } p+q \text { is odd }\end{cases} \\
& l k\left(T_{\left.e^{g_{\alpha}}, T_{f^{g_{\alpha}}}+\right)}= \begin{cases}(d-1) l k\left(\bar{P}, \bar{P}^{+}\right)+l k\left(T_{e}^{c}, T_{f}^{c+}\right), & \text { if } p+q \text { is even } \\
(d-1) l k\left(\bar{P}, \bar{P}^{+}\right)+l k\left(T_{e}^{c}, T_{f}^{c+}\right)+\frac{1}{2} d, & \text { if } p+q \text { is odd },\end{cases} \right.
\end{aligned}
$$

where $T_{e_{0}^{g_{\alpha}}}$ consists of $d$ copies of $P$,

(2) if $e_{0} \in T_{f}$ and if $e_{0} \notin T_{e}$,

$$
l k\left(T_{f^{g_{\alpha}}}, T_{e^{g_{\alpha}}}{ }^{+}\right)=l k\left(T_{f}^{c}, T_{e}^{+}\right), l k\left(T_{e^{g_{\alpha}}}, T_{f^{g_{\alpha}}}{ }^{+}\right)=l k\left(T_{e}, T_{f}^{c+}\right),
$$

(3) if $e_{0} \notin T_{f}$ and if $e_{0} \in T_{e}$,

$$
l k\left(T_{f^{g_{\alpha}}}, T_{e^{g_{\alpha}}}{ }^{+}\right)=l k\left(T_{f}, T_{e}^{c+}\right), \quad l k\left(T_{e^{g_{\alpha}}}, T_{f^{g_{\alpha}}}{ }^{+}\right)=l k\left(T_{e}^{c}, T_{f}{ }^{+}\right),
$$


(4) if $e_{0} \notin T_{f}$ and $e_{0} \notin T_{e}$,

$$
l k\left(T_{f^{g_{\alpha}}}, T_{e^{g_{\alpha}}}{ }^{+}\right)=l k\left(T_{f}, T_{e}^{+}\right), \quad l k\left(T_{e^{g_{\alpha}}}, T_{f^{g_{\alpha}}}{ }^{+}\right)=l k\left(T_{e}, T_{f}^{+}\right) .
$$

Proof. Assume that $T_{e} \backslash\{e\}$ consists of $p_{e}$ positive crossings and $q_{e}$ negative crossings and $T_{f} \backslash\{e\}$ consists of $p_{f}$ positive crossings and $q_{f}$ negative crossings, and that $T_{e} \cap T_{f}$ consists of $p_{e f}$ positive crossings and $q_{e f}$ negative crossings.

(1) Since $e_{0} \in T_{e} \cap T_{f}, T_{f^{g_{\alpha}}} \cap T_{e^{g_{\alpha}}}$ consists of $d p-p_{e}-p_{f}+p_{e f}$ positive crossings and $d q-q_{e}-q_{f}+q_{e f}$ negative crossings. Note that $d$ is even because $T_{e_{0}^{g_{\alpha}}}$ is a closed curve which consists of $d$ copies of $P$, and that $p_{e}+q_{e}$ and $p_{f}+q_{f}$ are odd. If $p+q$ is even and if $p_{e f}+q_{e f}$ is even, $T_{f^{g_{\alpha}}} \cap T_{e^{g_{\alpha}}}$ consists of an even number of edges. By Theorem 2.2 and Lemma 5.6(1),

$$
\begin{aligned}
l k\left(T_{f^{g_{\alpha}}}, T_{e^{g_{\alpha}}}+\right) & =-\frac{1}{2}\left(\left(d p-p_{e}-p_{f}+p_{e f}\right)-\left(d q-q_{e}-q_{f}+q_{e f}\right)\right) \\
& =d \cdot l k\left(\bar{P}, \bar{P}^{+}\right)+l k\left(T_{f}, T_{e}^{+}\right)+\frac{1}{2}\left\{\left(p_{e}+p_{f}\right)-\left(q_{e}+q_{f}\right)\right\} \\
& =(d-1) l k\left(\bar{P}, \bar{P}^{+}\right)+l k\left(T_{f}^{c}, T_{e}^{c+}\right), \\
l k\left(T_{e^{g_{\alpha}}}, T_{f^{g_{\alpha}}}+\right) & =(d-1) l k\left(\bar{P}, \bar{P}^{+}\right)+l k\left(T_{e}^{c}, T_{f}^{c+}\right),
\end{aligned}
$$

because $l k\left(\bar{P}, \bar{P}^{+}\right)=-\frac{1}{2}(p-q)$.

If $p+q$ is even and if $p_{e f}+q_{e f}$ is odd, $T_{f^{g_{\alpha}}} \cap T_{e^{g_{\alpha}}}$ consists of an odd number of edges so that one can apply Theorem 2.2 and Lemma 5.6(1) to get the required results.

If $p+q$ is odd and if $p_{e f}+q_{e f}$ is even, $T_{f^{g_{\alpha}}} \cap T_{e^{g_{\alpha}}}$ consists of an even number of edges, while if $p+q$ is odd and if $p_{e f}+q_{e f}$ is odd, $T_{f^{g_{\alpha}}} \cap T_{e^{g_{\alpha}}}$ consists of an odd number of edges. By Theorem 2.2 and Lemma 5.6(1), one can get the required results. Notice that $l k\left(\bar{P}, \bar{P}^{+}\right)=-\frac{1}{2}(p-q+1)$.

(2) Since $e_{0} \in T_{f}$ and if $e_{0} \notin T_{e}, T_{f^{g_{\alpha}}}$ and $T_{e^{g_{\alpha}}}$ contains $p_{e}$ positive crossings and $q_{e}$ negative crossings in common. Since $p_{e}+q_{e}$ is odd, by Theorem 2.2 and Lemma 5.6(1),

$$
\begin{aligned}
l k\left(T_{f^{g_{\alpha}}}, T_{e^{g_{\alpha}}}{ }^{+}\right) & =\frac{1}{2}\left(p_{e}-q_{e}-1\right)=l k\left(T_{f}^{c}, T_{e}^{+}\right), \\
l k\left(T_{e^{g_{\alpha}}}, T_{f^{g_{\alpha}}}{ }^{+}\right) & =\frac{1}{2}\left(p_{e}-q_{e}+1\right)=l k\left(T_{e}, T_{f}^{c+}\right) .
\end{aligned}
$$

(3) Similar with (2).

(4) Since $e_{0} \notin T_{f}$ and $e_{0} \notin T_{e}, T_{e^{g_{\alpha}}}$ and $T_{f^{g_{\alpha}}}$ are related as the same way with the relation between $T_{e}$ and $T_{f}$ in the base link, so that we have the results.

Lemma 5.8. For $\alpha, \beta$ with $k+1 \leq \alpha, \beta \leq n$,

$$
l k\left(T_{f^{g_{\alpha}}}, T_{e^{g_{\beta}}}{ }^{+}\right)=\left\{\begin{aligned}
0, & \text { if } \alpha \neq \beta ; \\
l k\left(T_{f}, T_{e}^{+}\right), & \text {if } \alpha=\beta ;
\end{aligned}\right.
$$


Proof. If $\alpha \neq \beta, T_{f^{g_{\alpha}}}$ and $T_{e^{g_{\beta}}}$ do not intersect so that $l k\left(T_{f^{g_{\alpha}}}, T_{e^{g_{\beta}}}{ }^{+}\right)=0$. If $\alpha=\beta, T_{e^{g_{\alpha}}}$ and $T_{f^{g_{\alpha}}}$ are related as the same way with the relation between $T_{e}$ and $T_{f}$ in the base link, so that $l k\left(T_{f^{g_{\alpha}}}, T_{e^{g_{\beta}}}{ }^{+}\right)=l k\left(T_{f}, T_{e}^{+}\right)$.

Lemma 5.9. Suppose that $e_{0} \in T_{e}$. For $\alpha, \beta$ with $k+1 \leq \alpha \leq n$ and $1 \leq \beta \leq k$,

$$
\begin{aligned}
& l k\left(T_{f^{g_{\alpha}}}, T_{e^{g_{\beta}}}{ }^{+}\right)= \begin{cases}-l k\left(T_{f}, T_{e}^{c+}\right), & \text { if } T_{f} g_{\alpha} \| T_{e^{g_{\beta}}} ; \\
l k\left(T_{f}, T_{e}^{c+}\right) & \text { if } T_{f^{g_{\alpha}}} \bigwedge \mid T_{e^{g_{\beta}}},\end{cases} \\
& l k\left(T_{e^{g_{\beta}}, T_{f^{g_{\alpha}}}}{ }^{+}\right)= \begin{cases}-l k\left(T_{e}^{c}, T_{f}^{+}\right), & \text {if } T_{f^{g_{\alpha}}} \| T_{e^{g_{\beta}}} ; \\
l k\left(T_{e}^{c}, T_{f}^{+}\right), & \text {if } T_{f^{g_{\alpha}}} \lambda \mid T_{e^{g_{\beta}}} .\end{cases}
\end{aligned}
$$

Here $T_{f^{g_{\alpha}}} \| T_{e^{g_{\beta}}}$ means $T_{f^{g_{\alpha}}}$ and $T_{e^{g_{\beta}}}$ have the parallel orientation, while $T_{f^{g_{\alpha}}} \not \mid T_{e^{g_{\beta}}}$ means $T_{f_{g_{\alpha}}}$ and $T_{e^{g_{\beta}}}$ have the opposite orientation.

Proof. Assume that $T_{f} \backslash\{f\}$ contains $p_{f}$ positive crossings and $q_{f}$ negative crossings, so that $T_{f^{g_{\alpha}}}$ and $T_{e^{g_{\beta}}}$ contain $p_{f}$ positive crossings and $q_{f}$ negative crossings. Suppose that $T_{f} g_{\alpha}$ and $T_{e^{g_{\beta}}}$ have the parallel orientation. Since $p_{f}+q_{f}$ is odd, by Theorem 2.2 and Lemma 5.6 (3),

$$
\begin{aligned}
l k\left(T_{f^{g_{\alpha}}}, T_{e^{g_{\beta}}}{ }^{+}\right) & =-\frac{1}{2}\left(p_{f}-q_{f}-1\right)=-l k\left(T_{f}, T_{e}^{c+}\right) \\
l k\left(T_{e^{g_{\beta}}}, T_{f^{g_{\alpha}}}{ }^{+}\right) & =-\frac{1}{2}\left(p_{f}-q_{f}+1\right)=-l k\left(T_{e}^{c}, T_{f}^{+}\right) .
\end{aligned}
$$

For the case that $T_{f^{g_{\alpha}}}$ and $T_{e^{g_{\beta}}}$ have the opposite orientation, one can use the similar argument to get the required equations.

Remark 5.10. The equalities in Lemma 5.9 are hold for the case that $e$ is the fixed edge $e_{0}$.

Lemma 5.11. For $\alpha, \beta$ with $\alpha \neq \beta, 1 \leq \alpha, \beta \leq k$, assume that $T_{e^{g_{\alpha}}} \cap T_{e^{g_{\beta}}}$ consists of $d$ copies of $P$. Suppose that $P$ contains $p$ positive crossings and $q$ negative crossings.

(1) if $p+q$ is even and if $T_{f^{g_{\alpha}}} \| T_{e^{g_{\beta}}}$,

$$
l k\left(T_{f^{g_{\alpha}}}, T_{e^{g_{\beta}}}{ }^{+}\right)=d \cdot l k\left(\bar{P}, \bar{P}^{+}\right), \quad l k\left(T_{e^{g_{\beta}}}, T_{f^{g_{\alpha}}}{ }^{+}\right)=d \cdot l k\left(\bar{P}, \bar{P}^{+}\right) .
$$

(2) if $p+q$ is even and if $T_{f^{g_{\alpha}}} \not \mid T_{e^{g_{\beta}}}$,

$$
l k\left(T_{f^{g_{\alpha}}}, T_{e^{g_{\beta}}}{ }^{+}\right)=-d \cdot l k\left(\bar{P}, \bar{P}^{+}\right), \quad l k\left(T_{e^{g_{\beta}}}, T_{f^{g_{\alpha}}}{ }^{+}\right)=-d \cdot l k\left(\bar{P}, \bar{P}^{+}\right) .
$$

(3) if $p+q$ is odd, $d$ is even and if $T_{f^{g_{\alpha}}} \| T_{e^{g_{\beta}}}$,

$$
l k\left(T_{f^{g_{\alpha}}}, T_{e^{g_{\beta}}}{ }^{+}\right)=d \cdot l k\left(\bar{P}, \bar{P}^{+}\right)+\frac{1}{2} d, \quad l k\left(T_{e^{g_{\beta}}}, T_{f^{g_{\alpha}}}{ }^{+}\right)=d \cdot l k\left(\bar{P}, \bar{P}^{+}\right)+\frac{1}{2} d .
$$


(4) if $p+q$ is odd, $d$ is even and if $T_{f^{g_{\alpha}}} \not \lambda T_{e^{g_{\beta}}}$,

$$
l k\left(T_{f^{g_{\alpha}}}, T_{e^{g_{\beta}}}{ }^{+}\right)=-d \cdot l k\left(\bar{P}, \bar{P}^{+}\right)-\frac{1}{2} d, \quad l k\left(T_{e^{g_{\beta}}}, T_{f^{g_{\alpha}}}{ }^{+}\right)=-d \cdot l k\left(\bar{P}, \bar{P}^{+}\right)-\frac{1}{2} d .
$$

(5) if $p+q$ is odd, $d$ is odd and if $T_{f^{g_{\alpha}}} \| T_{e^{g_{\beta}}}$,

$$
l k\left(T_{f^{g_{\alpha}}}, T_{e^{g_{\beta}}}{ }^{+}\right)=d \cdot l k\left(\bar{P}, \bar{P}^{+}\right)+\frac{1}{2}(d \pm 1), \quad l k\left(T_{e^{g_{\beta}}}, T_{f^{g_{\alpha}}}{ }^{+}\right)=d \cdot l k\left(\bar{P}, \bar{P}^{+}\right)+\frac{1}{2}(d \mp 1) .
$$

(6) if $p+q$ is odd, $d$ is odd and if $T_{f^{g_{\alpha}}} \not \mid T_{e^{g_{\beta}}}$,

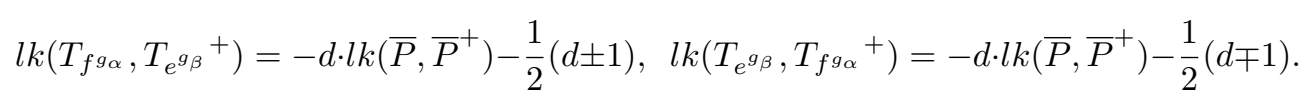

Proof. Since $T_{e^{g_{\alpha}}} \cap T_{e^{g_{\beta}}}$ consists of $d$ copies of $P, T_{f^{g_{\alpha}}} \cap T_{e^{g_{\beta}}}$ consists of $d p$ positive crossings and $d q$ negative crossings.

(1) Since $T_{f} g_{\alpha}$ and $T_{e^{g_{\beta}}}$ have the parallel orientation and since $d(p+q)$ is even, by Theorem 2.2,

$$
\begin{aligned}
& l k\left(T_{f^{g_{\alpha}}}, T_{e^{g_{\beta}}}{ }^{+}\right)=-\frac{1}{2}(d p-d q)=d \cdot l k\left(\bar{P}, \bar{P}^{+}\right), \\
& l k\left(T_{e^{g_{\beta}}}, T_{f^{g_{\alpha}}}{ }^{+}\right)=-\frac{1}{2}(d p-d q)=d \cdot l k\left(\bar{P}, \bar{P}^{+}\right),
\end{aligned}
$$

because $l k\left(\bar{P}, \bar{P}^{+}\right)=-\frac{1}{2}(p-q)$.

(2) Since $T_{f^{g_{\alpha}}}$ and $T_{e^{g_{\beta}}}$ have the opposite orientation, $-T_{f^{g_{\alpha}}}$ and $T_{e^{g_{\beta}}}$ have the parallel orientation, where $-T_{f^{g_{\alpha}}}$ denotes the reverse curve of a curve $T_{f^{g_{\alpha}}}$. By (1)

$$
\begin{aligned}
& l k\left(T_{f^{g_{\alpha}}}, T_{e^{g_{\beta}}}{ }^{+}\right)=-l k\left(-T_{f^{g_{\alpha}}}, T_{e^{g_{\beta}}}{ }^{+}\right)=-d \cdot l k\left(\bar{P}, \bar{P}^{+}\right), \\
& l k\left(T_{e^{g_{\beta}}, T_{f^{g_{\alpha}}}}{ }^{+}\right)=-l k\left(-T_{e^{g_{\beta}}}, T_{f^{g_{\alpha}}}{ }^{+}\right)=-d \cdot l k\left(\bar{P}, \bar{P}^{+}\right) .
\end{aligned}
$$

(3) Since $T_{f^{g_{\alpha}}}$ and $T_{e^{g_{\beta}}}$ have the parallel orientation and since $d(p+q)$ is even, by Theorem 2.2 ,

$$
\begin{aligned}
l k\left(T_{f^{g_{\alpha}}}, T_{e^{g_{\beta}}}{ }^{+}\right) & =-\frac{1}{2}(d p-d q)=d \cdot l k\left(\bar{P}, \bar{P}^{+}\right)+\frac{1}{2} d, \\
l k\left(T_{e^{g_{\beta}}}, T_{f^{g_{\alpha}}}{ }^{+}\right) & =-\frac{1}{2}(d p-d q)=d \cdot l k\left(\bar{P}, \bar{P}^{+}\right)+\frac{1}{2} d,
\end{aligned}
$$

because $l k\left(\bar{P}, \bar{P}^{+}\right)=-\frac{1}{2}(p-q+1)$.

(4) Similar with (2).

(5) Since $T_{f^{g_{\alpha}}}$ and $T_{e^{g_{\beta}}}$ have the parallel orientation and since $d(p+q)$ is odd, by Theorem 2.2 ,

$$
\begin{aligned}
& l k\left(T_{f^{g_{\alpha}}}, T_{e^{g_{\beta}}}{ }^{+}\right)=-\frac{1}{2}(d p-d q \pm 1)=d \cdot l k\left(\bar{P}, \bar{P}^{+}\right)+\frac{1}{2}(d \pm 1), \\
& l k\left(T_{e^{g_{\beta}}, T_{f^{g_{\alpha}}}+}\right)=-\frac{1}{2}(d p-d q \mp 1)=d \cdot l k\left(\bar{P}, \bar{P}^{+}\right)+\frac{1}{2}(d \mp 1),
\end{aligned}
$$


because $l k\left(\bar{P}, \bar{P}^{+}\right)=-\frac{1}{2}(p-q+1)$.

(6) Similar with (2).

Remark 5.12. The equalities in Lemma 5.11 are hold for the case that both $e$ and $f$ are the fixed edge $e_{0}$.

From Lemma 5.1, Lemma 5.3 and Lemma 5.7-Lemma 5.11, one can get the following theorem.

Theorem 5.13. Let $D$ be a diagram of a link with two fixed edges e and $f$, which is depicted as in Figure 8. If $\phi: E(D) \rightarrow G$ is a voltage assignment such that $\phi(g)=1_{G}$ for all edge $g \neq e, f$. A Seifert matrix of the symmetric link $D \times{ }_{\phi} G$ can be obtained from the Seifert matrix of $D$ and the information of the group action.

In the following example, we will use our lemmas to calculate a Seifert matrix of $D \times{ }_{\phi} G$ in Figure 9 .

Example 5.14. Recall that $G=\mathbb{Z}_{2} \oplus \mathbb{Z}_{2}$ and that the embedding surface $\widetilde{S}$ for $D \times{ }_{\phi} G$ is the 2 -sphere $S^{2}$. We have seen that Figure 11 shows the generators $\left\{T_{a_{1}}, T_{a_{2}}\right\}$ of $H_{1}(F)$ and the generators of $H_{1}(\widetilde{F})$ :

$$
\left\{T_{a_{1}^{g_{1}}}, T_{a_{2}^{g_{1}}}, T_{a_{1}^{g_{2}}}, T_{a_{2}^{g_{2}}}, T_{a_{1}^{g_{3}}}, T_{a_{2}^{g_{3}}}, T_{a_{1}^{g_{4}}}, T_{a_{2}^{g_{4}}}, T_{e_{0}^{g_{1}}}\right\} .
$$

One can see that our Seifert matrix for $D$ is given by

$$
M=\left(\begin{array}{rr}
-1 & -1 \\
0 & -1
\end{array}\right) .
$$

The Seifert matrix of $D \times_{\phi} G$ is obtained by using above lemmas, as seen in the following table.

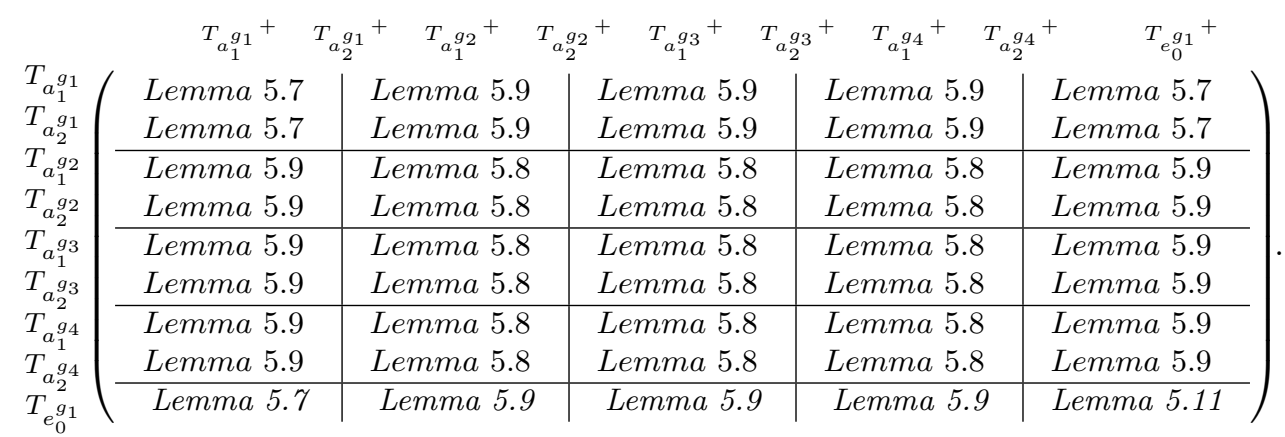

Notice that

$$
l k\left(\bar{P}, \bar{P}^{+}\right)=-\frac{1}{2}(1-0+1)=-1,
$$

because the path $P$ in this example has $p=1$ positive crossings and $q=0$ negative crossings. Since $T_{e_{0}^{g_{1}}}$ consists of 4 copies of $P, l k\left(T_{a_{1}^{g_{1}}}, T_{a_{1}^{g_{1}}}{ }^{+}\right)=3(-1)-1+2=-2$ 
by Lemma $5.7(1)$ and $l k\left(T_{e_{0}^{g_{1}}}, T_{e_{0}^{g_{1}}}{ }^{+}\right)=4 \cdot(-1)+\frac{1}{2} \cdot 4=-2$ by Lemma 5.11 . One can calculate the other entries by applying a suitable equation in the lemmas. Indeed, the Seifert matrix $\widetilde{M}$ for $D \times_{\phi} G$ is given by

$\left(\begin{array}{cc|cc|cc|cc|c}-2 & -2 & 0 & 0 & 0 & 0 & 0 & 0 & -2 \\ -1 & -2 & 0 & 0 & 0 & 0 & 0 & 0 & -2 \\ \hline-1 & -1 & -1 & -1 & 0 & 0 & 0 & 0 & -1 \\ -1 & -1 & 0 & -1 & 0 & 0 & 0 & 0 & -1 \\ \hline-1 & -1 & 0 & 0 & -1 & -1 & 0 & 0 & -1 \\ -1 & -1 & 0 & 0 & 0 & -1 & 0 & 0 & -1 \\ \hline 1 & 1 & 0 & 0 & 0 & 0 & -1 & -1 & 1 \\ 1 & 1 & 0 & 0 & 0 & 0 & 0 & -1 & 1 \\ \hline-1 & -1 & 0 & 0 & 0 & 0 & 0 & 0 & -2\end{array}\right)$

\section{References}

[1] Y. Choi, M. -J. Jeong and C. -Y. Park, Twist of knots and the $Q$-polynomials, Kyungpook Math. J., 44(2004), 449-467.

[2] S. Garoufalidis, Signatures of links and finite type invariants of cyclic branched covers, Tel Aviv Topology Conference: Rothenberg Festschrift, (1998), 87-97, Contemp. Math., 231, Amer. Math. Soc., Providence, RI, 1999.

[3] J. L. Gross and T. W. Tucker, Topological graph theory, John Wiley \& Sons, 1987.

[4] L. H. Kauffman and L. R. Taylor, Signature of links, Trans. Amer. Math. Soc., 216(1976), 351-365.

[5] A. Kawauchi, A survey of knot theory, Birkhäuser -Verlag,Basel, Boston, and Berlin, 1996.

[6] K. H. Ko and W. T. Song, Seifert matrices of periodic knots, J. Knot Theory Ramifications, 16(1)(2007), 45-57.

[7] S. Y. Lee, M. -S. Park and M. Seo, The Seifert matrices of periodic links with rational quotients, Kyungpook Math. J., 47(2)(2007), 295-309.

[8] J. Levine, The role of the Seifert matrix in knot theory, Actes du Congres International des Mathematiciens (Nice, 1970), Tome 2, pp. 95-98. Gauthier-Villars, Paris, 1971.

[9] W. Lickorish, An Introduction to Knot Theory, Springer-Verlag New York, Inc., 1997.

[10] Y. Miyazawa, Knots with a trivial coefficient polynomial, Kyungpook Math. J., 49(4)(2009), 801-809.

[11] K. Murasugi, On the signature of links, Topology 9, (1970), 283-298.

[12] H. Seifert, Über das geschlecht von knoten, Math. Ann, 110, 1934.

[13] H. F. Trotter, On S-equivalence of Seifert matrices, Invent. Math., 20(1973), 173-207.

[14] A. White, Graphs, Groups and Surfaces, Elsevier Science Publishers B.V, 1984. 